\title{
Genetic influences on nicotinic a5 receptor (CHRNA5) CpG methylation and mRNA expression in brain and adipose tissue
}

\author{
Jessica E. Ramsay ${ }^{1,2}$, C. Harker Rhodes ${ }^{3}$, Keerthi Thirtamara-Rajamani ${ }^{2}$ and Ryan M. Smith ${ }^{1,2^{*}}$
}

\begin{abstract}
Introduction: The nicotinic a5 receptor subunit, encoded by CHRNA5, harbors multiple functional single nucleotide polymorphisms (SNPs) that affect mRNA expression and alter the encoded protein. These polymorphisms are most notably associated with drug-taking behaviors and cognition. We previously identified common SNPs in a distant regulatory element (DRE) that increase CHRNA5 mRNA expression in the human prefrontal cortex (PFC) and confer risk for nicotine dependence. Genome-wide epigenetic studies in PFC and adipose tissue find strong effects of the DRE SNPs on CpG methylation. However, it is unclear whether DRE SNPs influence CpG methylation en route to modulating CHRNA5 mRNA expression. It is also unclear whether these polymorphisms affect expression in other brain regions, especially those mediating drug-taking behaviors.
\end{abstract}

Results: By measuring total and allelic CHRNA5 mRNA expression in human habenula and putamen autopsy tissues, we found that CHRNA5 DRE variants considerably increase mRNA expression by up to 3.5 -fold in both brain regions. Our epigenetic analysis finds no association between CpG methylation and CHRNA5 mRNA expression in the PFC or adipose tissues.

Conclusions: These finding suggests the mechanisms responsible for the genetic modulation of CpG methylation and mRNA expression are independent despite the DRE SNPs being highly associated with both measures. Our findings support a strong association between the DRE SNPs and mRNA expression or CpG methylation in the brain and periphery, but the independence of the two measures leads us to conclude that environmental factors affecting $\mathrm{CpG}$ methylation do not appear to directly modulate gene expression.

Keywords: Nicotinic receptors, Nicotine, mRNA expression, Enhancer, Repressor, Silencer, Methylation, Epigenetics, Expression quantitative trait loci, eQTL, Functional polymorphism, Allelic expression

\section{Introduction}

Previous studies found that allelic variation in the $\alpha 5 / \alpha 3 / \beta 4$ neuronal nicotinic acetylcholine receptor (nAChR) subunit gene cluster on chromosome region 15q25.1 significantly increases risk for addiction to multiple classes of drugs [1-9], but confers a protective effect for cocaine addiction $[8,10]$. This region also confers risk for lung cancer and chronic obstructive pulmonary disease (COPD) [2, 11-13]. A non-synonymous single nucleotide

\footnotetext{
* Correspondence: smith.4051@osu.edu

'Center for Pharmacogenomics, The Ohio State University, Columbus, OH 43210, USA

2Department of Pharmacology, The Ohio State University, 5184A Graves Hall, 333. W. 10th Ave., Columbus, OH 43210, USA

Full list of author information is available at the end of the article
}

polymorphism (SNP), rs16969968, in the gene encoding the $\alpha 5$ subunit (CHRNA5) is most commonly implicated in this gene cluster. Functional analysis of this SNP suggests it reduces ligand-mediated signaling [14, 15]. In addition to rs16969968 affecting protein function, SNPs in a cis-acting distal regulatory element (DRE), located $\sim 15$ $\mathrm{kb}$ upstream of CHRNA5, increase mRNA expression in the prefrontal cortex (PFC) up to 4-fold [9]. This DRE harbors a cluster of six SNPs (rs7164030, rs1979905, rs1979906, rs1979907, rs880395, and rs905740) in near complete linkage disequilibrium (LD). Joint analysis of rs880395 in the DRE with rs16969968 in the Collaborative Genetic Study of Nicotine Dependence (COGEND) finds increased risk for nicotine dependence compared to risk associated with either SNP alone [9], suggesting that both 
SNPs can influence phenotypes associated with this genomic region.

Knockout mouse studies examining the behavioral effects of habenular and ventral tegmental area (VTA) Chrna5 mRNA expression find that mice with a null mutation for Chrna5 significantly increase nicotine intake $[15,16]$ and exhibit attenuated nicotine-induced locomotion [17]. Re-expressing Chrna5 in the medial habenula $(\mathrm{MHb})$ reduces nicotine consumption to wildtype levels [16], suggesting that $\alpha 5 \mathrm{nAChR}$ mRNA expression in the $\mathrm{MHb}$ mediates negative reward signaling through the habenulo-interpenduncular pathway. Expression of the $\alpha 5$ receptor subunit in GABAergic neurons of the interpeduncular nucleus (IPN) was found to further modulate this $\mathrm{MHb}$ output to serotonergic brain regions [18]. The medial and lateral habenula are also connected to brain regions classically associated with drug-taking behaviors that express CHRNA5 mRNA. This includes afferent connections from the nucleus accumbens and efferent connections to the VTA and substantia nigra, which go on to innervate the PFC and striatum, respectively [19]. In the VTA, Chrna5 modulates the sensitivity of dopaminergic neurons to acute nicotine [15] but not ethanol administration [20]. Furthermore, rs16969968 interacts with a splicing SNP in the dopamine D2 receptor gene (DRD2), also implicated in addiction [21], to affect multiple aspects of prefrontal cortex physiology and behavior [22]. Together, these results demonstrate a pervasive functional profile for CHRNA5 in brain regions central to addiction and cognition. Despite strong evidence for altered Chrna5 expression in the rodent habenula affecting addiction phenotypes, and the association of regulatory DRE SNPs with nicotine addiction, it is unknown whether the DRE SNPs affect CHRNA5 mRNA expression in the human habenula. However, evidence that they modulate expression in the PFC, amygdala, and nucleus accumbens $[5,6,9,23]$, suggests the DRE exerts influence in cortical and subcortical brain regions.

CpG methylation in the CHRNA5 locus is strongly influenced by the DRE polymorphisms according to genome-wide scans of cis-methylation quantitative trait loci (cis-mQTLs) in the prefrontal cortex [24] and biopsied adipose [25] tissue. Moreover, specific CpG sites within the CHRNA5 promoter are hypermethylated in response to adverse childhood events [26]. These same adverse events confer risk for nicotine dependence, even exhibiting an genotype $x$ environment interaction specifically for rs16969968 [27]. While it is reasonably hypothesized that environmental factors affect methylation, which then influences expression, this relationship has not been formally tested. Thus, the relationship between genotype, methylation, and expression remains unclear and needs to be resolved in order to identify the mechanisms underlying substance abuse with respect to CHRNA5.
Here, we have tested the influence of the DRE variants modulate on CHRNA5 mRNA expression in the human habenula and putamen, by measuring total and allelic CHRNA5 mRNA expression. We also compared expression and CpG methylation across DRE genotypes using publicly available genome-wide datasets from BrainCloud [28], BrainCloudMethyl [24], and the Multiple Tissue Human Expression Resource [25, 29]. We find the DRE SNPs modulate expression in the putamen and habenula. However, in PFC and adipose tissues where we have measures of both expression and methylation from the same individuals, we find that methylation does not appear to directly influence CHRNA5 expression.

\section{Materials and methods \\ Tissue samples}

Twenty-one human habenula autopsy samples were dissected by a trained neuropathologist $(C R H)$ or obtained from the NICHD Brain and Tissue Bank for Developmental Disorders, while 57 human posterior putamen autopsy samples were obtained through the University of Miami Brain Endowment Bank. Demographics for these human tissues are presented in Table 1. Post-mortem tissue collection was performed in accordance with local Institutional Review Board approvals. The overall study described here was performed in accordance with the Institutional Review Board of The Ohio State University.

\section{Nucleic acid isolation \& complementary DNA (cDNA) synthesis}

Genomic DNA (gDNA) was isolated from all human tissues using a 'salting out' method adjusted for lipid-rich brain tissue, as previously described [9]. Total RNA was isolated by homogenizing the tissues in TRIzol and precipitating the RNA from the aqueous phase using isopropanol. We further purified the RNA using RNeasy Mini Kit spin columns (Qiagen, Germantown, MD) and digested latent gDNA on the column with recombinant DNaseI, as previously described [9]. cDNA preparations were made using $0.5 \mu \mathrm{g}$ total RNA for each sample. Gene-specific primers $(25 \mathrm{nM})$ supplemented with Oligo-dT $(5 \mu \mathrm{M})$ were used to prime the reverse transcription reaction.

\section{Sample genotyping}

SNPs rs16969968, rs615470, and rs7164030 were genotyped by restriction fragment length polymorphism (RFLP) methods. rs16969968 and rs615470 serve as marker SNPs for measuring allelic mRNA expression, which were chosen because of their high minor allele frequencies, high likelihood to be present in the mature mRNA, and LD pattern which suggests their minor alleles are present on different haplotypes. rs7164030 serves as the representative marker of DRE, which 
Table 1 Demographic characteristics for habenula and posterior putamen tissues

\begin{tabular}{llllllll}
\hline Tissue $(n)$ & Sex & Race $^{\text {a }}$ & Nicotine use & Cocaine use & Age (Avg. \pm S.D.) & PMI (Avg. \pm S.D.) & RIN (Avg. \pm S.D. \\
\hline Habenula (21) & Male: 12 & EA: 19 & Users: 5 & Users: N/A & $57.5 \pm 18.6$ & $35.6 \pm 38.0$ & \\
& Female: 9 & AA: 2 & Non-users: 7 & Non-users: N/A & & & \\
& & & Unknown: 9 & & & \\
Putamen (55) & Male: 46 & EA: 34 & Users: 24 & Users: 22 & $35.6 \pm 8.9$ & $16.7 \pm 5.4$ & N/A \\
& Female: 9 & AA: 10 & Non-users: 31 & Non-users: 33 & & \\
& & & & & \\
& & & & & \\
\end{tabular}

aEA: European-American, AA: African-American, Other: Mixed race and/or Hispanic

Abbreviations: PMI post-mortem interval, RIN RNA Integrity Number, as measured by Agilent Bioanalyzer 2100, N/A data not available

include 5 additional SNPs in high LD (rs1979907, rs19 79906, rs1979905, rs880395, and rs905740). The gDNA regions surrounding these SNPs were amplified using primers tagged with a fluorophore (6-FAM or HEX) and the resultant amplicons were cut with restriction enzymes (rs16969968-Taq1a; rs615470-CviQI; rs7164030-Tsp509I) that recognize only one of the two alleles resulting from the presence of the polymorphism. Fragments were resolved on an ABI 3730 DNA Analyzer (Life Technologies) or by standard gel electrophoresis (1.5\% agarose).

\section{Total and allelic mRNA expression measurement}

Total CHRNA5 and $\beta$-actin $(A C T B)$ mRNA expression was measured in all human and mouse tissues by qPCR using an ABI 7500 Fast Sequence Detection System (Life Technologies). In addition, we measured the expression of two highly-enriched habenula markers, POU4F1 and CHRNB3, in the habenula samples to determine the purity of the dissections. The relative quantity of CHRNA5 mRNA was normalized within each sample to $A C T B$ mRNA expression for statistical analysis. The influences of available covariates (age, sex, race, post-mortem interval, RNA integrity number, nicotine use, or habenula purity) were tested on $A C T B$-normalized total CHRNA5 mRNA expression in each brain region using stepwise linear regression.

We quantified allelic mRNA expression in habenula and putamen samples heterozygous for rs16969968 or rs615470 using a fluorescent primer extension method (SNaPshot), as previously described [9]. Fluorescentlylabeled primer extension fragments, representing the two different alleles of rs16969968 or rs615470, were resolved on an ABI 3730. The fluorescent peak heights for each allele, determined using GeneMapper 4.0 Software (Life Technologies), were used to calculate relative allelic expression ratios (ancestral/variant allele). For each sample, at least two separate measurements were used to calculate allelic expression imbalance (AEI). Allelic ratios for cDNA were normalized against the overall average ratio calculated for gDNA for each marker SNP. We subsequently compared the absolute magnitude of the allelic expression in samples heterozygous for rs7164030 versus homozygotes for either allele.

\section{CHRNA5 mRNA expression and CpG methylation using BrainCloud and MuTHER}

We utilized existing repositories of transcriptome-wide mRNA expression, genome-wide methylation profiles, and SNP genotypes in postnatal PFC (BrainCloud; http://brain cloud.jhmi.edu/) and adipose tissue (MuTHER; http://www .muther.ac.uk/) to test interactions between DRE SNPs, expression, and CpG methylation. Detailed methods for these studies are available in their primary publications $[24,25,28,29]$. Source data can be obtained from the Gene Expression Omnibus (Accession: GSE30272), dbGAP (Accession: phs000417), EMBL-EBI ArrayExpress (Accessions: E-TABM-1140 and E-MTAB-1866), and by applying to the TwinsUK Consortium (http://www.twinsuk.ac.uk/).

Briefly, samples were genotyped with a variety of Illumina arrays (HumanHap300, HumanHap610Q, Human Hap650Y, Human 1 M-Duo, and Human 1.2 MDuo $1 \mathrm{M}$ ) and imputed to 1000 Genomes populations using IMPUTE2. From the imputed data, we used rs7164030 as a surrogate marker of the DRE to test genetic effects on expression and methylation. Samples were also measured for genome-wide $\mathrm{CpG}$ methylation, using the Infinium HumanMethylation450k BeadChip assay, and transcriptome-wide mRNA expression, using Illumina 49 K Oligo Arrays (BrainCloud) or HumanHT-12 v3 BeadChips (MuTHER). For analyses, we used CpG probes cg22563815 and cg17108064, which measures methylation at CpG sites 913 and 802 nucleotides upstream of the annotated CHRNA5 gene (hg19 chr15: 78856949 and chr15:78857060), respectively. Although these CpG probes are $\sim 12 \mathrm{~kb}$ downstream from the DRE SNPs, they are among the highest scoring mQTLs for the DRE SNPs in the CHRNA5 gene region. For expression, we used probes hHC002196 (BrainCloud) and ILMN_1770044 (MuTHER), which hybridize to CHRNA5 mRNA in the $3^{\prime}$ untranslated region and exon 5 , respectively. 


\section{Statistical analyses}

Statistical analyses were performed in $R$ (x64 v.3.1.0) with standardized $\beta$-coefficients calculated by the QuantPsyc package. For all datasets, we used interquartile range (IQR) to exclude extreme outliers, defined as data points below $Q 1-3 x I Q R$ or above $Q 3+3 x I Q R$. Next, we identified significant covariates using stepwise linear regression and AIC, using the [step] function to reach a minimal adequate model. We subsequently included significant covariates in analyses of rs7164030 genotype on expression and methylation or as interaction terms in linear regression models of expression and methylation. Potential covariates in our putamen and habenula expression datasets included age, sex, race, smoking history, cocaine use, and post-mortem interval. Potential covariates in the BrainCloud data included sex, age, race, and an estimate of neuron enrichment that was specific to methylation data [30]. Age and batch-specific effects were considered as a potential covariate in the MuTHER dataset. We tested for an overall effect of methylation on expression using linear regression across the entire BrainCloud or MuTHER sample populations and report the standardized $\beta$-coefficient for the methylation measure. We further tested this relationship within each rs7164030 genotype group to identify any genotype-specific effect that could be obscured when examining the population as a whole.

\section{Results}

Total and allelic CHRNA5 mRNA expression in putamen and habenula

Two putamen samples were excluded from analyses due to poor RNA quality as indicated by $A C T B$ expression $>2$ standard deviations above the mean of the remaining samples, leaving 55 total putamen samples. Stepwise linear regression revealed sex as a significant covariate of CHRNA5 mRNA expression measured via qPCR in the putamen. We found a significant effect of the representative DRE SNP rs7164030 genotype on putamen expression ( $n=55, F=28.90, p=1.82 \times 10^{-6}$; Fig. 1), whereby homozygous minor " $G$ " allele samples expressed 3.5-fold more CHRNA5 mRNA than homozygous major "A" allele samples, consistent with our previous findings in PFC [9]. Examining the influence of rs7164030 on habenular CHRNA5 mRNA expression via qPCR with race as a significant covariate revealed no significant effect of genotype, although the direction of the genotypic effect is consistent with our findings in the putamen and PFC. We also noted that the purity of the habenula dissection, as determined by POU4F1 or CHRNB3 expression, did not influence CHRNA5 mRNA expression. A comparative analysis of habenula CHRNA5 mRNA expression with previously measured PFC expression found no enrichment in the habenula relative to the PFC in humans, consistent

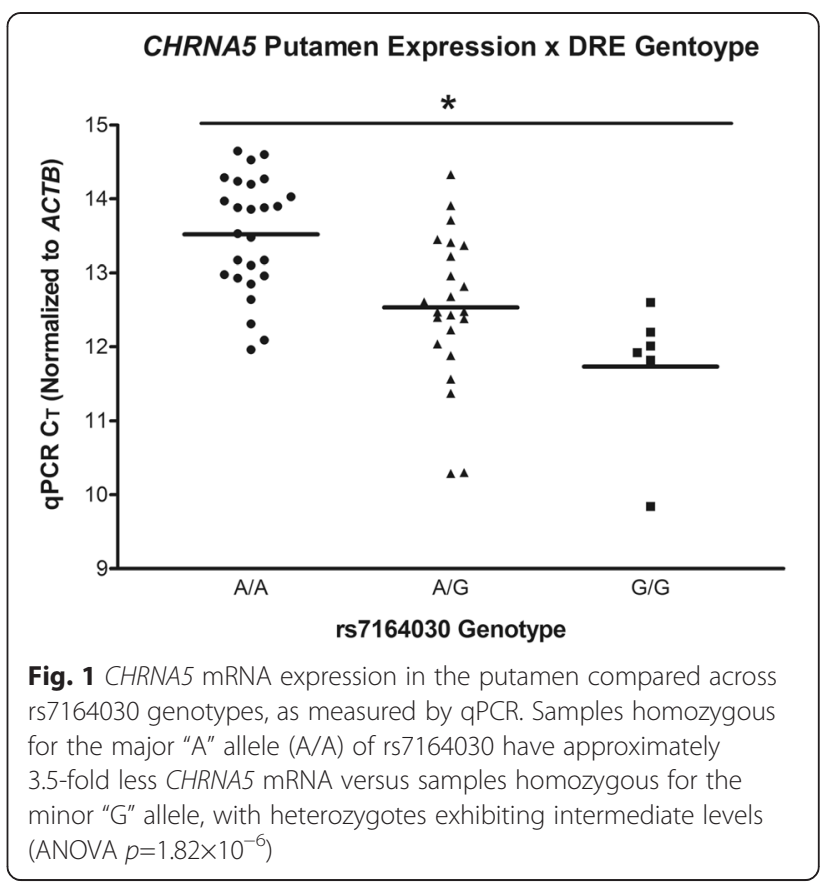

with previous reports of generally low expression in these areas in rodents $[18,31]$.

We measured allelic mRNA expression in 21 of 55 putamen samples heterozygous for either rs16969968 or rs615470 (9 co-heterozygous) and 9 of 21 habenula samples heterozygous for rs16969968. The low expression of CHRNA5 in both tissues required us to average the allelic ratio measurements at the two marker SNPs, as done for the putamen, or take an increased number of measurements at the same SNP, as done for the habenula. Thus, the averaged data is only presented for the 9 coheterozygous putamen samples, while the data for all habenula samples is presented for marker SNP rs16969968. Samples heterozygous for rs7164030 exhibited AEI ranging from 2.1 to 6.5-fold differences between the expression of the two alleles, while samples homozygous for either allele of rs7164030 all displayed AEI of <2fold, consistent across both brain regions. We observed greater expression for the major allele of rs16969968 relative to the minor allele in all but one sample exhibiting $>2$-fold AEI, consistent with the major allele for rs16969968 residing on the high expressing DRE haplotype. Comparing the absolute magnitude of AEI across rs7164030 genotype, we find heterozygotes exhibit significantly greater AEI versus homozygotes $(F=7.99, p=0.012$; Fig. 2), supporting the hypothesis that the DRE SNPs exert their function in both the habenula and putamen.

\section{DRE SNPs, CHRNA5 expression, and methylation in BrainCloud and MuTHER}

CHRNA5 mRNA expression differed significantly across rs7164030 genotype in the BrainCloud dataset $(F=28.57$, 


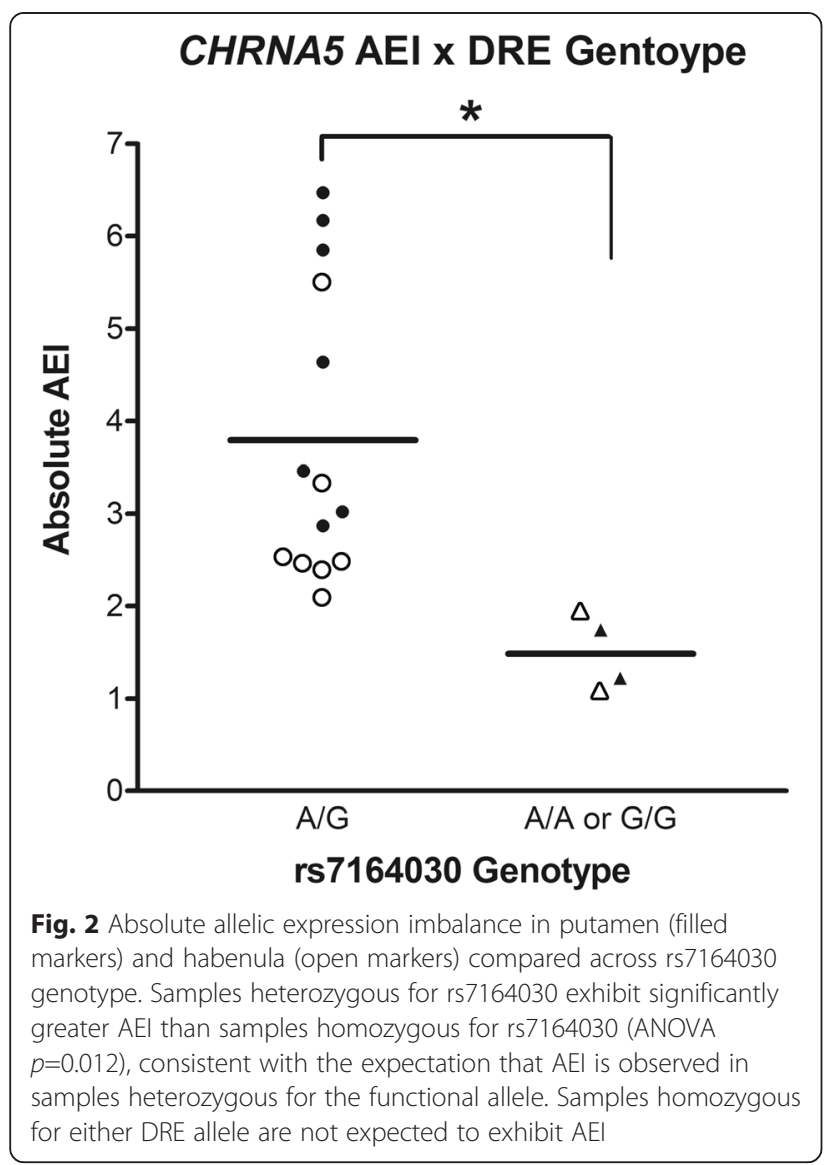

$p=2.30 \times 10^{-7}$; age and sex included as significant covariates). Consistent with our previous study in the PFC [9], samples homozygous for the variant $G$ allele of rs7164030 expressed 3.67-fold more CHRNA5 mRNA than samples homozygous for the ancestral A allele (Fig. 3a). rs7164030 genotype was also significantly associated with CHRNA5 expression in MuTHER $(F=13.06$, $p=3.28 \times 10^{-4}$; Fig. 3b).

Methylation in the BrainCloud dataset significantly differed across rs7164030 genotype in the PFC for both CpG probes (cg22563815: $F=109.17, p=5.87 \times 10^{-21}$; race, age, and neuron enrichment estimate as significant covariates; cg17108064: $F=191.06, p=1.77 \times 10^{-31}$; race as a significant covariate). Here, samples homozygous for the variant $G$ allele of rs7164030 had significantly greater CpG methylation at both probes relative to the ancestral A allele homozygotes (Fig. $3 \mathrm{c}$ and e). Methylation was also significantly higher across both probes for the variant $G$ allele carriers in the MuTHER dataset (cg22563815: $F=$ $1703.48, p=5.54 \times 10^{-172}$; bisulfite conversion gDNA concentration and efficiency included as covariates; cg17108064: $F=614.33, p=3.73 \times 10^{-92}$; batch and bisulfite conversion efficiency included as covariates; Fig. $3 \mathrm{~d}$ and $\mathrm{f}$ ).

Because the DRE SNPs are associated with significant increases in both expression and methylation, we further asked if the increased expression can be predicted by greater methylation using linear regression. In both the BrainCloud and MuTHER datasets, we found that methylation at either probe did not significantly predict CHRNA5 expression. The linear model for cg22563815 in BrainCloud (Expression sex + cg22563815*methylation"race"age"neuron count estimate"rs7164030) found no significant effect for methylation at $\operatorname{cg} 22563815(t=$ 0.42, $p=0.67 \beta=0.30$; Fig. 4a). Similarly, the model for cg17108064 (Expression $\sim$ sex + age + cg17108064* race*rs7164030) also revealed no significant effect for methylation ( $t=-0.84, p=0.40 \beta=-0.11$; Fig. $4 \mathrm{~b})$. In the MuTHER dataset, the linear model for methylation at cg22563815 (Expression cg22563815*bisulfite conversion concentration*bisulfite conversion efficiency*rs7164030) showed a trend towards significance $(t=-1.95, p=0.051, \beta=$ -2.34; Fig. 4c), while methylation at cg17108064 (Expression $\sim$ cg17108064*batch*bisulfite conversion efficiency* rs7164030) did not predict expression ( $t=1.48, p=0.14$, $\beta=0.99$; Fig. $4 \mathrm{~d}$ ).

Evident in each of our linear models is the confounding influence of genotype on both methylation and expression (Fig. 4). Although we accounted for this influence statistically in the model examining all samples, we subsequently tested whether it was still possible for methylation to influence expression on specific genetic backgrounds (i.e. if one were to carry the DRE SNPs), which could be obscured in the full linear model. Thus, we performed linear regression within each of the rs7164030 genotype groups, finding no evidence that methylation at either probe affects expression in any of the genetic backgrounds defined by the DRE SNPs (Table 2).

\section{Discussion}

Our findings reveal pervasive influence of the DRE SNPs on CHRNA5 mRNA expression and methylation in brain and adipose tissue, whereby the minor DRE alleles are associated with greater expression and methylation. This genotypic difference is consistent with our findings in the PFC for both the BrainCloud dataset and in our previous study [9]. The habenula did not show a main effect of the DRE SNPs on total CHRNA5 expression, but we observed strong allelic differences in the habenula that perfectly correlate with the DRE SNPs, consistent with the interpretation that they modulate CHRNA5 expression in the habenula. While significant, the influence of the DRE SNPs on CHRNA5 expression is not as strong in adipose tissue. We previously reported no influence of the DRE SNPs in lymphoblastoid cell lines (LCLs), but other studies with larger sample sizes have found cis-eQTLs for CHRNA5, implicating the DRE SNPs in peripheral whole blood [32], monocytes [33], and lung [34]. Thus, it is likely that the DRE SNPs are modulating 


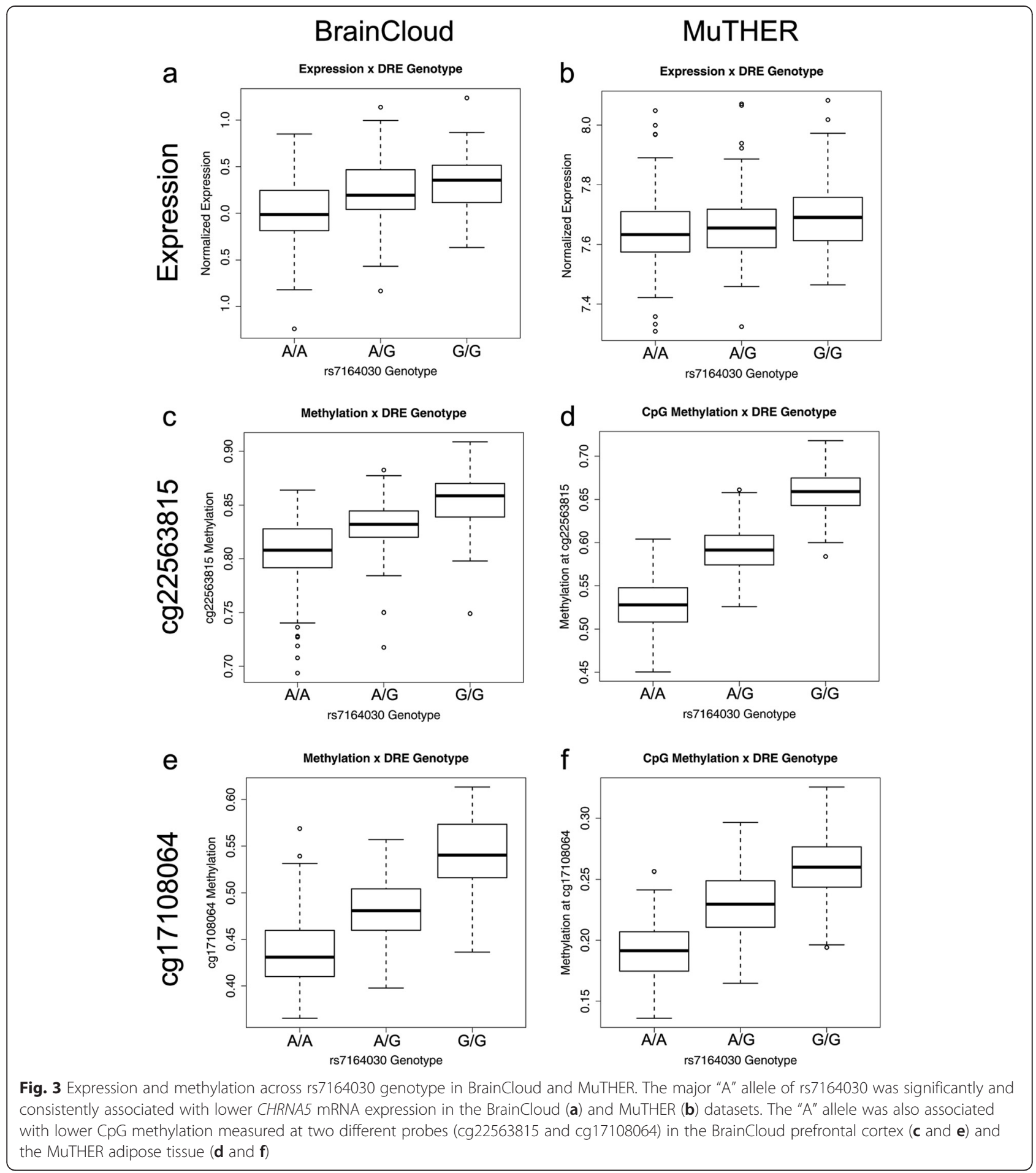

expression of CHRNA5 in peripheral tissues, but exert less influence relative to their impact in the brain.

The location and epigenetic histone markings in the CHRNA5 locus harboring the DRE SNPs previously led us to propose they act in an enhancer [9]. Data from the ENCyclopedia Of DNa Elements (ENCODE) Project [35] viewed on the UCSC Genome Browser [36] shows histone modifications in a lymphocyte cell line (GM12878) consistent with enhancers, including histone 3 lysine 4 monomethylation (H3K4Me1) and light trimethylation (H3K4Me3), and $\mathrm{H} 3$ lysine 27 acetylation (H3K27Ac). However, when a portion of the DRE containing rs880395, rs905740, and rs7164030 was sub-cloned into a vector upstream of a minimal promoter, it acted as a repressor, with 


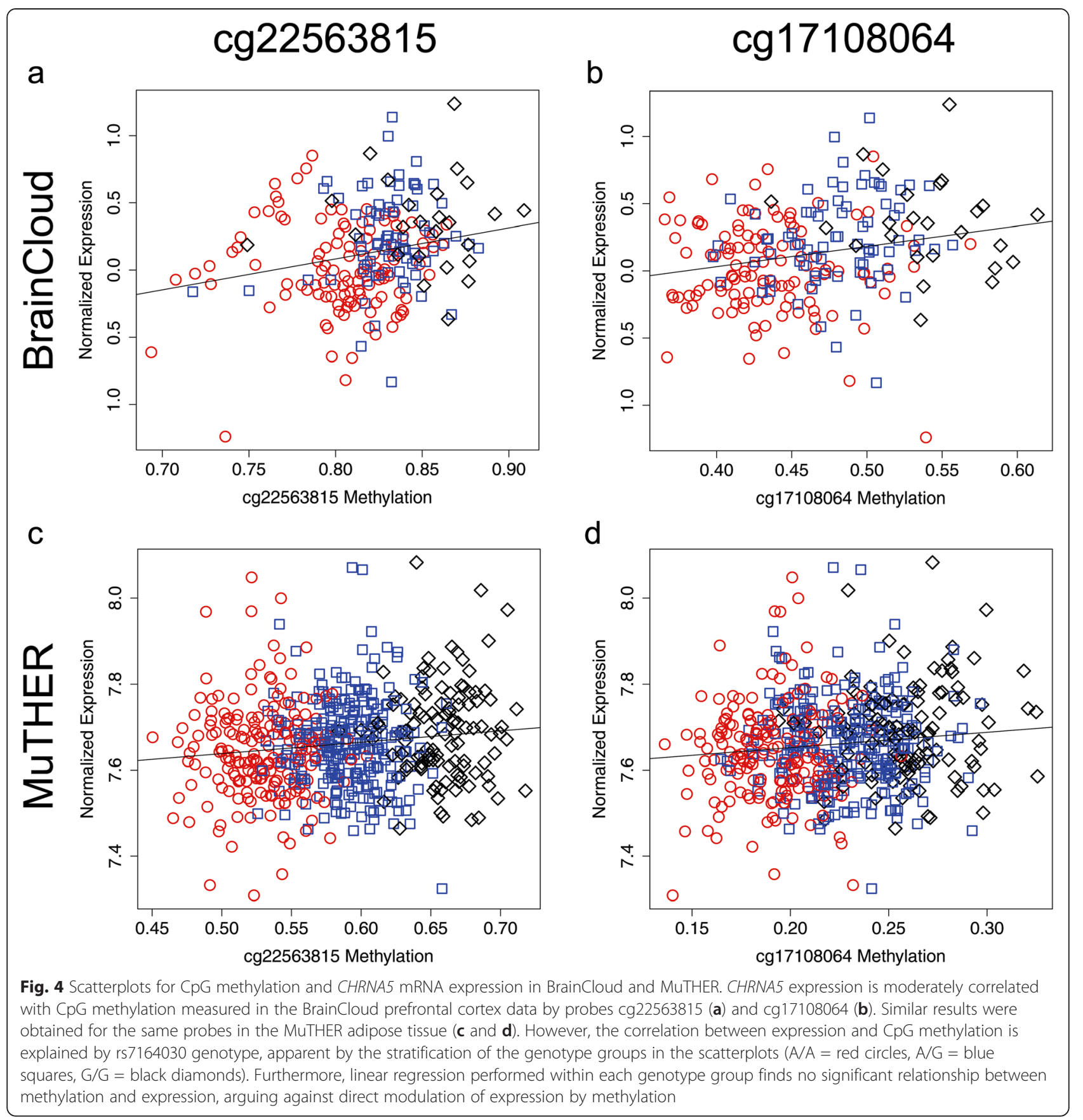

no significant expression differences between DRE haplotypes [23]. Given these contradictory results, we find it possible that the DRE contains both enhancer and repressor elements. A dual enhancer/repressor mechanism is not novel. Perhaps the most well-known example is the RE-1 Silencing Transcription Factor (REST), which silences neuronal genes in the periphery [37], but has the ability to enhance gene expression in the brain [38, 39]. Evolutionary studies of cis-acting enhancer elements supports the possibility that multiple variants affecting enhancer function can arise together within a population to high frequency [40], sometimes co-opting cryptic or existing regulatory sequences to derive their new functions [41], as we would assume occurred for the DRE SNPs in CHRNA5. A more thorough analysis of the evolutionary constraints on the CHRNA5 locus could provide clues about the adaptive evolution of regulatory elements in humans.

In addition to the epigenetic histone modifications present in the CHRNA5 locus, CpG methylation is strongly associated with CHRNA5 SNPs. Since CpG methylation can repress transcription [42] it led us to examine CHRNA5 CpG methylation and mRNA expression using BrainCloud 
Table 2 Linear regression results for CpG methylation and CHRNA5 gene expression across rs7164030 genotypes

\begin{tabular}{|c|c|c|c|c|c|}
\hline Dataset & CpG probe & rs7164030 genotype (n) & Standardized $\beta$ coefficient for methylation & $t$-value & $p$-value \\
\hline \multirow[t]{6}{*}{ BrainCloud } & cg22563815 & A/A (118) & 0.267 & 0.363 & 0.717 \\
\hline & $\operatorname{cg} 22563815$ & A/G (77) & 1.166 & 0.390 & 0.698 \\
\hline & cg22563815 & G/G (25) & -16.837 & -0.397 & 0.700 \\
\hline & cg17108064 & A/A (118) & -0.171 & -1.424 & 0.157 \\
\hline & $\operatorname{cg} 17108064$ & $A / G(77)$ & 0.153 & 1.072 & 0.288 \\
\hline & cg17108064 & G/G (25) & 0.024 & -0.040 & 0.969 \\
\hline \multirow[t]{6}{*}{ MUTHER } & cg22563815 & A/A (184) & 4.069 & 0.528 & 0.598 \\
\hline & cg22563815 & $A / G(270)$ & 0.010 & 0.014 & 0.989 \\
\hline & $\operatorname{cg} 22563815$ & $\mathrm{G} / \mathrm{G}(111)$ & -2.964 & -1.718 & 0.089 \\
\hline & $\operatorname{cg} 17108064$ & A/A (184) & 0.981 & 0.398 & 0.691 \\
\hline & $\operatorname{cg} 17108064$ & $\mathrm{~A} / \mathrm{G}(270)$ & -1.288 & -0.813 & 0.417 \\
\hline & cg17108064 & $\mathrm{G} / \mathrm{G}(111)$ & -2.510 & -0.639 & 0.524 \\
\hline
\end{tabular}

and MuTHER. Because the DRE SNPs were strongly associated with increased methylation and expression in both datasets, we expected methylation to be positively correlated with expression, thus providing mechanistic evidence linking epigenetic modulation of the CHRNA5 locus and gene expression. Instead, we found that methylation and expression were independent when accounting for DRE genotype and other significant covariates. Evidence that CHRNA5 expression and methylation are independent is important for delineating mechanisms underlying drug addiction that is associated with this gene locus, since both methylation and expression apparently influence addiction risk. One explanation that unifies expression and methylation, that also serves as a caveat of this study, is that methylation influences the expression of CHRNA5 in a way that was not detected here. Such scenarios could include changes in alternative splicing or transcription start site usage which do not change the overall levels of CHRNA5 mRNA, but alter the makeup of the mRNA. The GENCODE project has annotated an alternatively spliced transcript (ENST00000559554.1), but it has only been observed as an expressed sequence tag in a neuroblastoma cell line.

Methylation at the CHRNA5 locus is sensitive to environmental factors, as demonstrated by childhood adverse events (CA). CA results in hypermethylation and increased risk for drug dependence [26, 27]. Males carrying rs16969968 who experience CA are at greater risk for dependence relative to those without rs16969968 [27]. In the context of our findings, we do not find it likely that hypermethylation changes mRNA expression, although we cannot rule out that CA-induced hypermethylation can be much greater than observed in our samples and subsequently affect expression. Reanalyzing existing CA studies to include the DRE SNPs in addition to rs16969968 could shed some light on the relationships between CA, methylation, and smoking risk. However, a study examining environmental factors, CpG methylation, mRNA expression, and drug dependence would be ideal for resolving the risk conferred by $15 \mathrm{q} 25.1$.

Finally, the strong impact of Chrna5 expression in mouse $\mathrm{MHb}$ and VTA on nicotine consumption despite low levels of mRNA expression in mice and humans signifies the importance of the specific cell types on which these receptors are expressed. The $\mathrm{MHb}$ afferents that express the $\alpha 5$ subunit project to the interpeduncular nucleus [19], which also contains GABAergic neurons expressing the $\alpha 5$ subunit [18], modulating aversiveness associated with nicotine intake [16] and withdrawal [43]. Dopaminergic neurons in the VTA express the $\alpha 5$ receptor subunit [44] and project to multiple addictionrelated brain regions, including the cortex and insula via the mesocortical pathway and limbic areas, the nucleus accumbens, lateral habenula, and amygdala through the mesolimbic pathway [45]. Finding ways to modulate the firing of the cells expressing CHRNA5, directly or indirectly, without targeting nicotinic $\alpha 5$-containing receptors could provide avenues for treating addictive behaviors that circumvent the inherent challenges of developing small molecules for nicotinic receptors. Identifying promising new targets will require a firm understanding of addiction neurocircuitry and of genetic expression within specific cell types in the habenula, IPN, and VTA, in order to exploit the aversive signaling properties of these cells in the context of drug abuse.

\section{Conclusions}

Our findings support pervasive but independent influence of the CHRNA5 DRE SNPs on mRNA expression and CPG methylation in the brain and periphery. With evidence that environmental influences modulate $\mathrm{CpG}$ methylation in this region, we advocate for future studies to incorporate environmental, epigenetic, and genetic factors in the pathogenesis of addiction associated with 
CHRNA5. Finally, clinical and molecular genetic studies of the CHRNA5/A3/B4 locus suggest the presence of unaccounted for functional regulatory variants [46], requiring additional studies to delineate the exact functional variant(s) and their phenotypic impact to further disentangle disease risk conferred by $15 \mathrm{q} 25.1$.

\section{Competing interests}

The authors declare no competing financial interests related to the work described here.

\section{Authors' contributions}

JER performed studies, wrote and revised the manuscript. CHR dissected tissue, designed studies, and revised the manuscript. KTR performed studies and revised the manuscript. RMS designed studies, analyzed data, wrote and revised the manuscript. All authors read and approved the final manuscript.

\section{Acknowledgements}

The authors thank Dr. Elin Grundberg at McGill University and Dr. Andrew Jaffe at the Lieber Institute for Brain Development for their help in accessing and analyzing the MUTHER and BrainCloud datasets.

\section{Funding and disclosure}

This study was supported by a grant from the US National Institutes of Health, General Medical Sciences, U01 GM092655.

MUTHER via TwinsUK: The study was funded by the Wellcome Trust; European Community's Seventh Framework Programme (FP7/2007-2013). The study also receives support from the National Institute for Health Research (NIHR) - funded BioResource, Clinical Research Facility and Biomedical Research Centre based at Guy's and St Thomas' NHS Foundation Trust and King's College London. SNP Genotyping was performed by The Wellcome Trust Sanger Institute and National Eye Institute via NIH/CIDR

\section{Author details}

${ }^{1}$ Center for Pharmacogenomics, The Ohio State University, Columbus, $\mathrm{OH}$ 43210, USA. ${ }^{2}$ Department of Pharmacology, The Ohio State University, 5184A Graves Hall, 333. W. 10th Ave., Columbus, OH 43210, USA. ${ }^{3}$ National Institute of Mental Health, Human Brain Collection Core, 10 Center Drive, Rm. 4N306, Bethesda, MD, USA.

Received: 2 March 2015 Accepted: 13 July 2015

Published online: 01 October 2015

\section{References}

1. Saccone NL, Culverhouse RC, Schwantes-An TH, Cannon DS, Chen X, Cichon S, et al. Multiple independent loci at chromosome 15q25.1 affect smoking quantity: A meta-analysis and comparison with lung cancer and COPD. PLOS Genet. 2010;6, e1001053.

2. Saccone SF, Hinrichs AL, Saccone NL, Chase GA, Konvicka K, Madden PA, et al. Cholinergic nicotinic receptor genes implicated in a nicotine dependence association study targeting 348 candidate genes with 3713 SNPs. Hum Mol Gen. 2007;16:36-49.

3. Berrettini W, Yuan X, Tozzi F, Song K, Francks C, Chilcoat H, et al. Alpha-5/ alpha-3 nicotinic receptor subunit alleles increase risk for heavy smoking. Mol Psychiatry. 2008;13:368-73.

4. Schlaepfer IR, Hoft NR, Collins AC, Corley RP, Hewitt JK, Hopfer CJ, et al. The CHRNA5/A3/B4 gene cluster variability as an important determinant of early alcohol and tobacco initiation in young adults. Biol Psychiatry. 2008:63:1039-46.

5. Wang JC, Cruchaga C, Saccone NL, Bertelsen S, Liu P, Budde JP, et al. Risk for nicotine dependence and lung cancer is conferred by mRNA expression levels and amino acid change in CHRNA5. Hum Mol Gen. 2009;18:3125-35.

6. Wang JC, Grucza R, Cruchaga C, Hinrichs AL, Bertelsen S, Budde JP, et al. Genetic variation in the CHRNA5 gene affects mRNA levels and is associated with risk for alcohol dependence. Mol Psychiatry. 2009;14:501-10.

7. Erlich PM, Hoffman SN, Rukstalis M, Han JJ, Chu X, Linda Kao WH, et al. Nicotinic acetylcholine receptor genes on chromosome $15 q 25.1$ are associated with nicotine and opioid dependence severity. Hum Genet. 2010;128:491-9.
8. Sherva R, Kranzler HR, Yu Y, Logue MW, Poling J, Arias AJ, et al. Variation in nicotinic acetylcholine receptor genes is associated with multiple substance dependence phenotypes. Neuropsychopharmacology. 2010;35:1921-31.

9. Smith RM, Alachkar H, Papp AC, Wang D, Mash DC, Wang JC, et al. Nicotinic alpha5 receptor subunit mRNA expression is associated with distant $5^{\prime}$ upstream polymorphisms. Eur J Hum Genet. 2011:19:76-83.

10. Grucza RA, Wang JC, Stitzel JA, Hinrichs AL, Saccone SF, Saccone NL, et al. A risk allele for nicotine dependence in CHRNA5 is a protective allele for cocaine dependence. Biol Psychiatry. 2008;64:922-9.

11. Amos Cl, Wu X, Broderick P, Gorlov IP, Gu J, Eisen T, et al. Genome-wide association scan of tag SNPs identifies a susceptibility locus for lung cancer at 15q25.1. Nat Genet. 2008;40:616-22.

12. Hung RJ, McKay JD, Gaborieau V, Boffetta P, Hashibe M, Zaridze D, et al. A susceptibility locus for lung cancer maps to nicotinic acetylcholine receptor subunit genes on 15q25. Nature. 2008;452:633-7.

13. Wilk JB, Shrine NR, Loehr LR, Zhao JH, Manichaikul A, Lopez LM, et al. Genome-wide association studies identify CHRNA5/3 and HTR4 in the development of airflow obstruction. Am J Respir Crit Care Med. 2012;186:622-32

14. Bierut LJ, Stitzel JA, Wang JC, Hinrichs AL, Grucza RA, Xuei X, et al. Variants in nicotinic receptors and risk for nicotine dependence. Am J Psychiatry. 2008;165:1163-71.

15. Morel C, Fattore L, Pons S, Hay YA, Marti F, Lambolez B, et al. Nicotine consumption is regulated by a human polymorphism in dopamine neurons. Mol Psychiatry. 2014;19:930-6.

16. Fowler CD, Lu Q, Johnson PM, Marks MJ, Kenny PJ. Habenular alpha5 nicotinic receptor subunit signalling controls nicotine intake. Nature. 2011:471:597-601.

17. Salas R, Orr-Urtreger A, Broide RS, Beaudet A, Paylor R, De Biasi M. The nicotinic acetylcholine receptor subunit alpha 5 mediates short-term effects of nicotine in vivo. Mol Pharmacol. 2003;3:1059-66.

18. Hsu YW, Tempest L, Quina LA, Wei AD, Zeng H, Turner EE. Medial habenula output circuit mediated by alpha5 nicotinic receptor-expressing gabaergic neurons in the interpeduncular nucleus. J Neurosci. 2013;33:18022-35.

19. Bianco $H$, Wilson SW. The habenular nuclei: a conserved asymmetric relay station in the vertebrate brain. Philos Trans R Soc Lond B Biol Sci. 2009;364:1005-20.

20. Chatterjee S, Santos N, Holgate J, Haass-Koffler CL, Hopf FW, Kharazia V, et al. The a5 subunit regulates the expression and function of $a 4^{*}$-containing neuronal nicotinic acetylcholine receptors in the ventral-tegmental area. PLoS One. 2014;8, e68300.

21. Moyer RA, Wang D, Papp AC, Smith RM, Duque L, Mash DC, et al. Intronic polymorphisms affecting alternative splicing of human dopamine D2 receptor are associated with cocaine abuse. Neuropsychopharmacology. 2011;36:753-62.

22. Di Giorgio A, Smith RM, Fazio L, D’Ambrosio E, Gelao B, Tomasicchio A, et al. DRD2/CHRNA5 interaction on prefrontal biology and physiology during working memory. PLoS One. 2014;9, e95997.

23. Doyle GA, Wang MJ, Chou AD, Oleynick JU, Arnold SE, Buono RJ, et al. In vitro and ex vivo analysis of chrna3 and chrna5 haplotype expression. PLoS One. 2011;6, e23373.

24. Numata S, Ye T, Hyde TM, Guitart-Navarro X, Tao R, Wininger M, et al. DNA methylation signatures in development and aging of the human prefrontal cortex. Am J Hum Genet. 2012;90:260-72.

25. Grundberg E, Meduri E, Sandling JK, Hedman ÅK, Keildson S, Buil A, et al. Global analysis of DNA methylation variation in adipose tissue from twins reveals links to disease-associated variants in distal regulatory elements. Am J Hum Genet. 2013:93:876-90.

26. Zhang H, Wang F, Kranzler HR, Zhao H, Gelernter J. Profiling of childhood adversity-associated DNA methylation changes in alcoholic patients and healthy controls. PLoS One. 2013;8, e65648.

27. Xie $\mathrm{P}$, Kranzler HR, Zhang H, Oslin D, Anton RF, Farrer LA, et al. Childhood adversity increases risk for nicotine dependence and interacts with alpha5 nicotinic acetylcholine receptor genotype specifically in males. Neuropsychopharmacology. 2012;37:669-76.

28. Colantuoni C, Lipska BK, Ye T, Hyde TM, Tao R, Leek JT, et al. Temporal dynamics and genetic control of transcription in the human prefrontal cortex. Nature. 2011;478:519-23.

29. Grundberg E, Small KS, Hedman ÅK, Nica AC, Buil A, Keildson S, et al. Mapping cis and trans regulatory effects across multiple tissues in twins. Nat Genet. 2012:44:1084-9. 
30. Guintivano J, Aryee MJ, Kaminsky ZA. A cell epigenotype specific model for the correction of brain cellular heterogeneity bias and its application to age, brain region and major depression. Epigenetics. 2013;8:290-302.

31. Han ZY, Le Novere N, Zoli M, Hill Jr JA, Champtiaux N, Changeux JP. Localization of nAChR subunit mRNAs in the brain of macaca mulatta. Eur J Neurosci. 2000;12:3664-74.

32. Westra HJ, Peters MJ, Esko T, Yaghootkar H, Schurmann C, Kettunen J, et al. Systematic identification of trans eQTLs as putative drivers of known disease associations. Nat Genet. 2013;45:1238-43.

33. Zeller T, Wild P, Szymczak S, Rotival M, Schillert A, Castagne R, et al. Genetics and beyond - The transcriptome of human monocytes and disease susceptibility. PLoS One. 2010;5, e10693.

34. Hao K, Bossé Y, Nickle DC, Paré PD, Postma DS, Laviolette M, et al. Lung eQTLs to help reveal the molecular underpinnings of asthma. PLoS Genet. 2012;8, e1003029.

35. Rosenbloom KR, Sloan CA, Malladi VS, Dreszer TR, Learned K, Kirkup VM, et al. Encode data in the UCSC genome browser: Year 5 update. Nucleic Acids Res. 2013;41:D56-63.

36. Kent WJ, Sugnet CW, Furey TS, Roskin KM, Pringle TH, Zahler AM, et al. The human genome browser at UCSC. Genome Res. 2002;12:996-1006.

37. Schoenherr CJ, Anderson DJ. The neuron-restrictive silencer factor (NRSF): A coordinate repressor of multiple neuron-specific genes. Science. 1995;267:1360-3.

38. Bessis A, Champtiaux N, Chatelin L, Changeux JP. The neuron-restrictive silencer element: A dual enhancer/silencer crucial for patterned expression of a nicotinic receptor gene in the brain. Proc Natl Acad Sci U S A. 1997;94:5906-11.

39. Seth KA, Majzoub JA. Repressor element silencing transcription factor/ neuron-restrictive silencing factor (REST/NRSF) can act as an enhancer as well as a repressor of corticotropin-releasing hormone gene transcription. J Biol Chem. 2001;276:13917-23.

40. Rebeiz M, Pool JE, Kassner VA, Aquadro CF, Carroll SB. Stepwise modification of a modular enhancer underlies adaptation in a drosophila population. Science. 2009;326:1663-7.

41. Rebeiz M, Jikomes N, Kassner VA, Carroll SB. Evolutionary origin of a novel gene expression pattern through co-option of the latent activities of existing regulatory sequences. Proc Natl Acad Sci U S A. 2011;108:10036-43.

42. Curradi M, Izzo A, Badaracco G, Landsberger N. Molecular mechanisms of gene silencing mediated by DNA methylation. Mol Cell Biol. 2002;22:3157-73.

43. Salas R, Sturm R, Boulter J, De Biasi M. Nicotinic receptors in the habenulointerpeduncular system are necessary for nicotine withdrawal in mice. J Neurosci. 2009;29:3014-8.

44. Klink R, de Kerchove d'Exaerde A, Zoli M, Changeux JP. Molecular and physiological diversity of nicotinic acetylcholine receptors in the midbrain dopaminergic nuclei. J Neurosci. 2001;21:1452-63.

45. Russo SJ, Nestler EJ. The brain reward circuitry in mood disorders. Nat Rev Neurosci. 2013;14:609-25.

46. Schwantes-An TH, Culverhouse R, Duan W, Ramnarine S, Rice JP, Saccone $\mathrm{NL}$. Interpreting joint SNP analysis results: When are two distinct signals really two distinct signals? Genet Epidemiol. 2013;37:301-9.

\section{Submit your next manuscript to BioMed Central and take full advantage of:}

- Convenient online submission

- Thorough peer review

- No space constraints or color figure charges

- Immediate publication on acceptance

- Inclusion in PubMed, CAS, Scopus and Google Scholar

- Research which is freely available for redistribution

Submit your manuscript at www.biomedcentral.com/submit 